Eric Schuster, Christoph Strümpfel, Svenja Huschbeck, Bastian Göbel, Christian Berth, Maarten Uijt De Haag

\title{
Precise Relative Navigation and Separation Assurance of UAS and Manned Aircraft during Low Altitude Airfield Operations
}

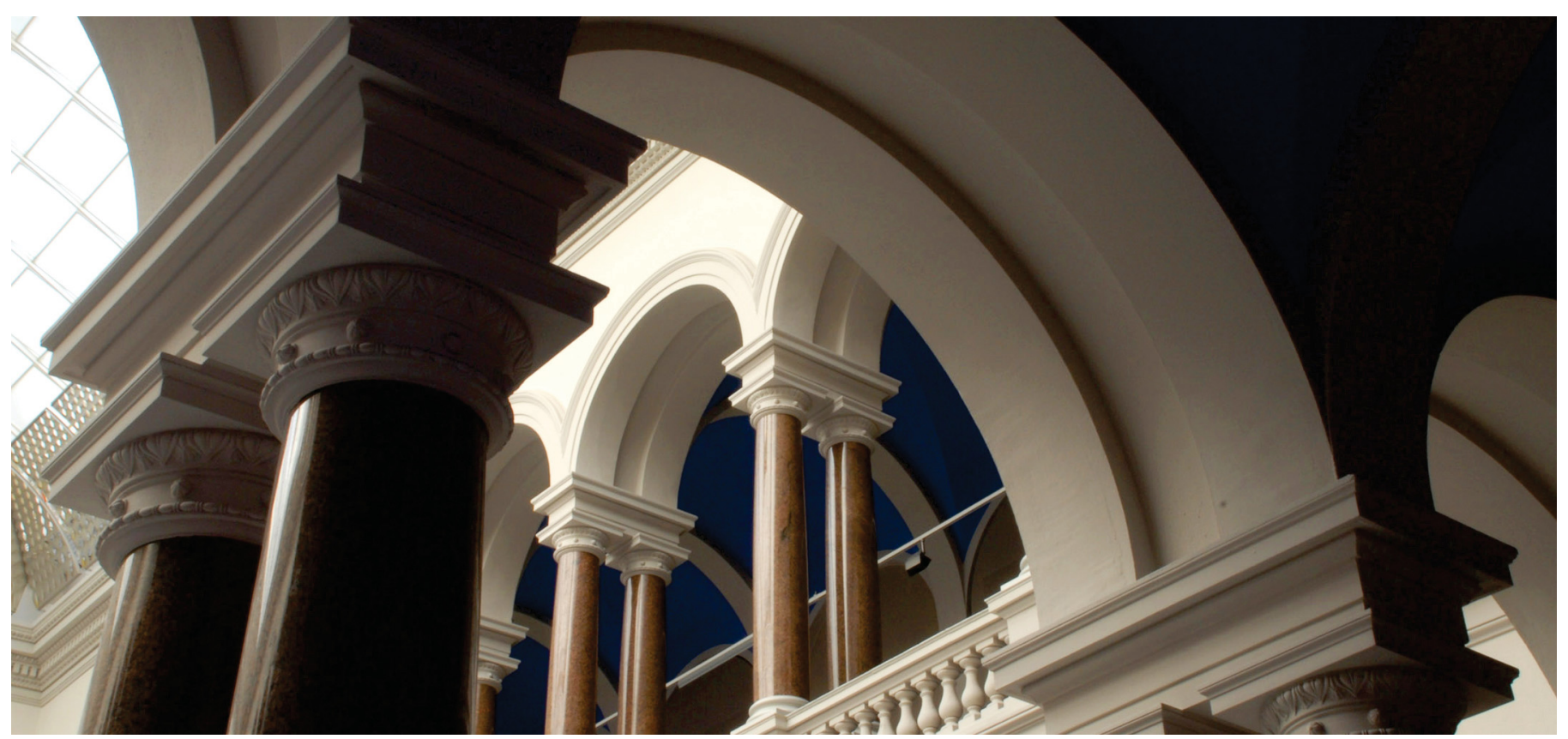

Schuster, E., Strümpfel, C., Huschbeck, S., Göbel, B., Berth, C., \& Uijt De Haag, M. (2020). Precise Relative Navigation and Separation Assurance of UAS and Manned Aircraft during Low Altitude Airfield Operations. AIAA Scitech 2020 Forum. Presented at the AIAA Scitech 2020 Forum. https://doi. org/10.2514/6.2020-0057 


\title{
Precise Relative Navigation and Separation Assurance of UAS and Manned Aircraft during Low Altitude Airfield Operations
}

\author{
Eric Schuster ${ }^{1}$, Christoph Strümpfel ${ }^{1}$, Svenja Huschbeck ${ }^{1}$, Bastian Göbel ${ }^{2}$, Christian Berth ${ }^{2}$, \\ and Maarten Uijt de $\mathrm{Haag}^{3}$ \\ Technische Universität Berlin
}

This paper was first published in the proceedings of the AIAA SciTech Forum 2020 in Orlando and does not show any changes in content to the primary publication. The full reference to the first publication is:

Eric Schuster, Christoph Struempfel, Svenja Huschbeck, Bastian Goebel, Christian Berth and Maarten Uijt De Haag (2020). Precise Relative Navigation and Separation Assurance of UAS and Manned Aircraft during Low Altitude Airfield Operations. AIAA Scitech 2020 Forum. https://doi.org/10.2514/6.2020-0057

\begin{abstract}
This paper discusses the flight test setup and preliminary results of a precise relative navigation and separation assurance system to support safe simultaneous operation of general aviation aircraft and UAS at low altitudes in the vicinity of an uncontrolled airfield. With an increased use of UAS for a wide variety of applications, such as law enforcement, search and rescue, agriculture, infrastructure inspection, maintenance, mapping, filming and journalism, it is expected that UAS will be taking off, landing or otherwise operating at airfields at the same time as manned aircraft. In that case, it will be required that the UAS not only has precise knowledge of its location and velocity to enable an automatic flight at lower altitudes with sufficient accuracy, integrity, availability and continuity to support its operation (e.g. take-off, landing, flight patterns), but also to support broadcasting its position and velocity to the other aircraft in the vicinity, for improved awareness of the UAS location and to achieve separation assurance
\end{abstract}

\section{NOMENCLATURE}

\begin{tabular}{|c|c|c|c|c|c|}
\hline CPA & $=$ & Closest Point of Approach & $t$ & $=$ & time \\
\hline$r$ & $=$ & Vectoral distance & $\mathrm{dt}$ & $=$ & timestep \\
\hline$\dot{r}$ & $=$ & Rate of change in range & $\mathrm{t}_{\mathrm{CPA}}$ & $=$ & time to Closest Point of Approach \\
\hline$S_{x}$ & $=$ & $\mathrm{x}$-component of the slant range & $T_{\mathrm{CPA}}$ & $=$ & time to Closest Point of Approach \\
\hline $\mathrm{S}_{\mathrm{y}}$ & $=$ & $y$-component of the slant range & TMOD & $=$ & modified time to Closest Point of \\
\hline$S_{z}$ & $=$ & z-component of the slant range & & & Approach \\
\hline & $\begin{array}{l}= \\
=\end{array}$ & $\begin{array}{l}\text { Ground speed } \\
\text { Distance at Closest }\end{array}$ & VUAS & $=$ & Ground speed of unmanned aerial \\
\hline & & Approach & & & Ground speed of aircraft \\
\hline
\end{tabular}

\footnotetext{
Research Assistant and PhD candidate, Chair of Flight Guidance \& Air Transport, TU Berlin. Research Assistant and flight test pilot, Chair of Flight Guidance \& Air Transport, TU Berlin. Professor of Chair, Chair of Flight Guidance \& Air Transport, TU Berlin, AIAA Associate Fellow.
} 


\section{INTRODUCTION}

This paper discusses the flight test setup and preliminary results of a precise relative navigation and separation assurance system to support safe simultaneous operation of general aviation aircraft and UAS at low altitudes in the vicinity of an uncontrolled airfield. With an increased use of UAS for a wide variety of applications, such as law enforcement, search and rescue, agriculture, infrastructure inspection, maintenance, mapping, filming and journalism, it is expected that UAS will be taking off, landing or otherwise operating at airfields at the same time as manned aircraft. In that case, it will be required that the UAS not only has precise knowledge of its location and velocity to enable an automatic flight at lower altitudes with sufficient accuracy, integrity, availability and continuity to support its operation (e.g. take-off, landing, flight patterns), but also to support broadcasting its position and velocity to the other aircraft in the vicinity, for improved awareness of the UAS location and to achieve separation assurance. The latter will enable neighboring manned and unmanned aircraft, to perform collision avoidance maneuvres if a potential hazardous conflict situation develops. In this project, the team at the TU Berlin has developed a navigation and separation assurance unit for two UAS platforms and one manned aircraft based on algorithms developed by the TU Berlin and is preparing a flight demonstration in which these UAS and a manned aircraft (TU Berlin's LASER aircraft), operate in the same low-level airspace near an airfield (Eberswalde-Finow, ICAO: EDAV) north of Berlin, Germany. The operational concept is shown in Figure 1.

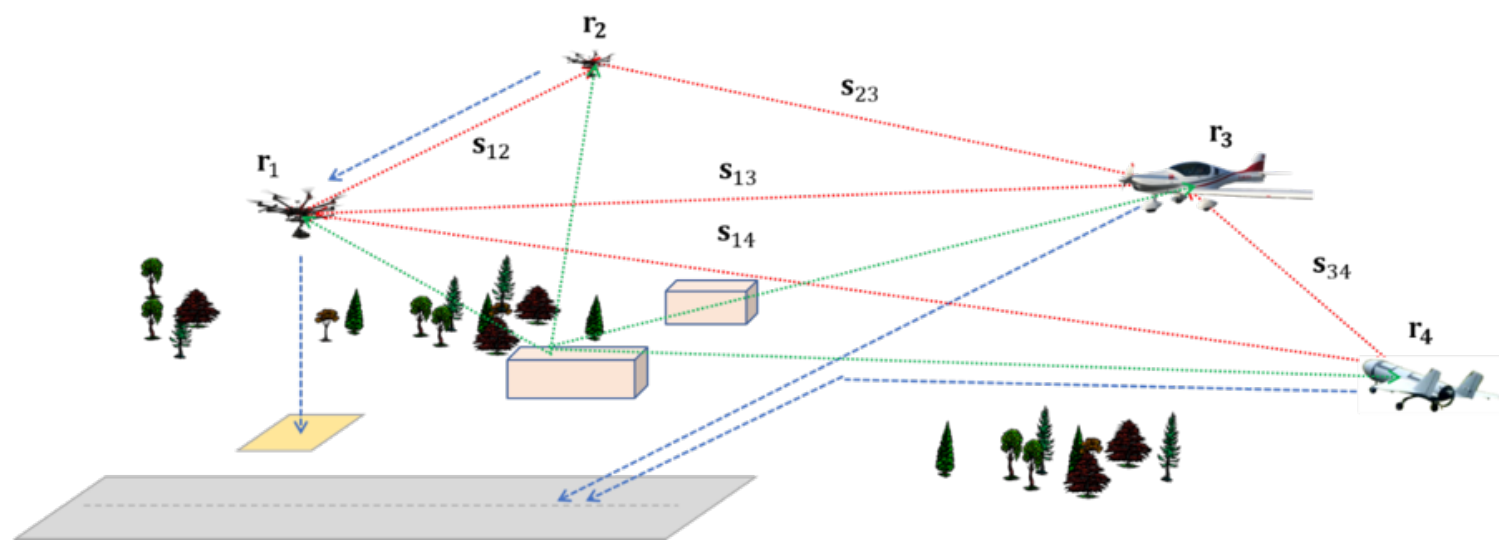

Fig. 1. Operational scenario for the flight test with multiple UAS and one manned aircraft operating in the same low-level airspace in the vicinity of an airfield.

As part of these operations, both, the manned aircraft and the teleoperated UAS can perform approach procedures while maintaining safe separation. In parallel, a second UAS is performing an agricultural mapping mission in the vicinity of the airport. Throughout all these procedures, position and velocity state information is exchanged between all participants and a ground station to enable conflict detection and collision avoidance (i.e. separation assurance).

With adoption of the flight test with regards to this general approach due to operational and legal reasons in the near vicinity of airfield within the Federal Republic of Deutschland the UAS's are imitated by a walking individual and a car. Both reflect different kinds of UAS in terms of their physical limitation. 


\section{METHODOLOGY - SEPARATION ASSURANCE}

The simultaneous operation of multiple air vehicles, manned and unmanned, both, in a segregated or unsegregated airspace implies possible hazardous situations in terms of violation of separation. As already done in controlled airspaces with manned aircrafts and high-performance UAS [1] a separation logic has to be implemented to assure safe operation to airspace users.

In general, collision avoidance can be expressed using three criteria. Since time to threat is more important than distance [2], especially when two objects move with high speeds, the T-criterion is applied. Furthermore, the range, altitude and bearing must be considered as available from the Traffic Collision and Avoidance System (TCAS) in larger aircraft [3], or the position and velocity reports as available from Automatic Dependent Surveillance - Broadcast (ADS-B). All these parameters vary as a function of time. The visualization in Figure 2 displays a possible hazardous scenario of an UAS and an aircraft moving in the horizontal (upper portion) as well as the vertical plane (lower portion).

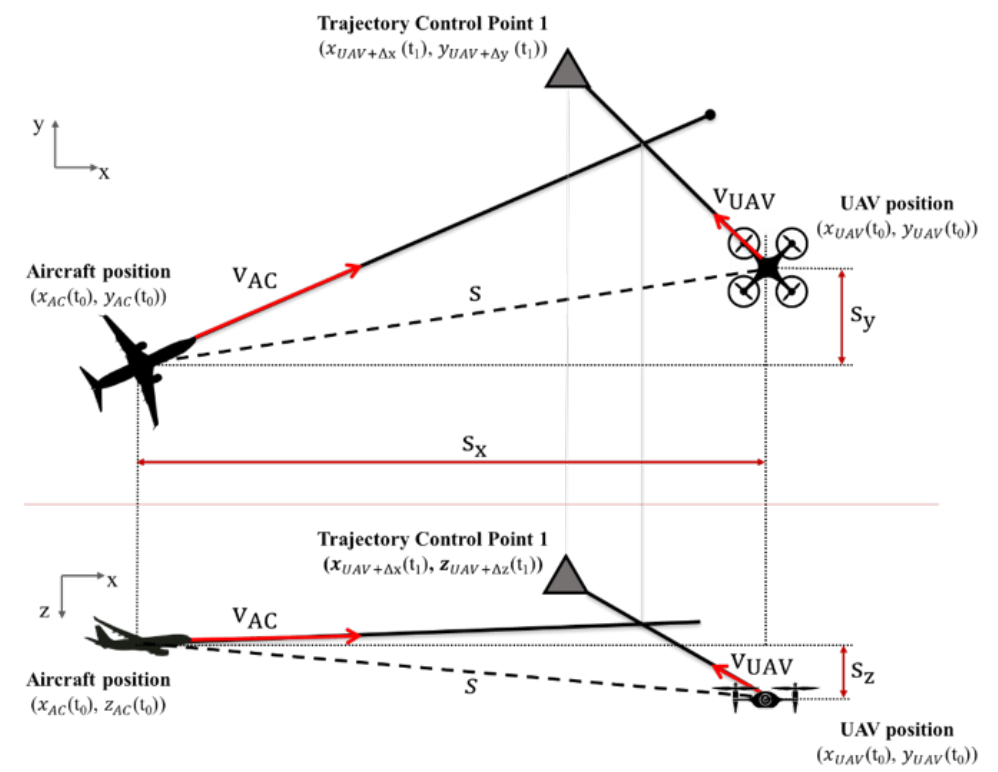

Fig. 2. Design CPA scenario with a manned and unmanned vehicle

The position of both vehicles can be described with their respective coordinates in $x, y$ and $z$ at time $t_{0}$. The separation vector that connects both aircraft is referred to as $\mathbf{s}$ in its composition in $s_{x}$, $s_{y}$ and $s_{z}$. The UAS flies towards the Trajectory Control Point 1 using the displayed flight path (solid line) with the speed of $\mathbf{v}_{U A V}$. At the same time, the aircraft velocity is given by $\mathbf{v}_{A C}$. can be interpreted. Hence, the relative velocity is given by $\mathbf{v}=\mathbf{v}_{A C}-\mathbf{v}_{U A V}$. The influence of wind is neglected for simplification purposes. Assuming only linear motion (i.e. acceleration is 0 ), the separation develops over time as:

$$
\mathbf{s}+t \mathbf{v}
$$

Mathematically, the value of the $\mathrm{T}$ at the closest point of approach (CPA) can obtained by minimizing the mean squared separation:

$$
(\mathbf{s}+t \mathbf{v})^{T}(\mathbf{s}+t \mathbf{v})
$$


Minimization of Eq.2 results in:

$$
\begin{gathered}
\frac{d}{d t}(\mathbf{s}+t \mathbf{v}) \cdot(\mathbf{s}+t \mathbf{v})=0 \Rightarrow 2 \mathbf{s} \cdot \mathbf{v}+2 t \mathbf{v} \cdot \mathbf{v}=\mathbf{0} \Rightarrow \\
2 \mathbf{s} \cdot \mathbf{v}+2 t \mathbf{v} \cdot \mathbf{v}=0
\end{gathered}
$$

This directly leads to

$$
t_{C P A}=-\frac{\mathbf{v} \cdot \mathbf{s}}{\mathbf{v} \cdot \mathbf{v}}=-\frac{\mathbf{v} \cdot \mathbf{s}}{\|\mathbf{v}\|^{2}}
$$

Replace the respective t-term in Eq. 1 returns the separation vector at $t_{C P A}$

$$
\mathbf{s}+t_{C P A} \mathbf{v}=\mathbf{s}-\frac{\mathbf{v} \cdot \mathbf{s}}{\|\mathbf{v}\|^{2}} \mathbf{v}
$$

And the corresponding miss-distance:

$$
d_{c p a}=\left\|\mathbf{s}+t_{C P A} \mathbf{v}\right\|
$$

Alternatively, the time to CPA can be used using the range $(r=\|\mathbf{s}\|)$ between both vehicles. With the rate of range change $\dot{r}$ given by:

$$
\dot{r}=\frac{\mathbf{s} \cdot \mathbf{v}}{r}
$$

Resulting in a time to CPA expressed in vectoral notation as:

$$
\tau_{C P A}=-\frac{r}{\dot{r}}=-\frac{\|\mathbf{s}\|^{2}}{\mathbf{s} \cdot \mathbf{v}}
$$

As shown through Eq. 3 this approach is just available for non-misses at the CPA. So, Eq. 8 must be adopted for actual miss-cases, since these cases occur more often. Thus, this approach does not account low closure rates or variations in acceleration. This can lead to two objects move towards each other down to small distances without raising an advisory alert, because the $\tau_{C P A}$-criterion is not violated until it is too late. So, a distance measure (DMOD) is added and modifies the T-criterion to this certain extend, such that the explained reduction in slant range can be detected.

$$
\tau_{C P A}=-\frac{\|\mathbf{s}\|^{2}}{\mathbf{s} \cdot \mathbf{v}}+\frac{D M O D^{2}}{\mathbf{s} \cdot \mathbf{v}}
$$

In addition to the horizontal component, the system also checks the altitude difference as well as a possible time to co-altitude. Depending on the application, the temporal and spatial components (DMOD) of Eq. 9 can be adapted. TCAS II [3] uses the above equations and defines values for DMOD and $\tau$ for different altitudes and alert level (i.e. traffic advisory or resolution alert). Currently, Detect and Avoid (DAA) systems for larger UAS operating in the same airspace as manned aircraft use a well clear definition basically evaluates the horizontal $(\|\mathbf{s}\|)$ and vertical $\left(\left|s_{Z}\right|\right)$ well clear [1] [4].

Equations (1) through (9), may require revision when operating in close vicinity at airfields. Furthermore, in that case the quality of the estimates for $\mathbf{s}$ and $\mathbf{v}$ are important. Examples, of how to obtain these accurate estimates as well as results of the above calculation of CPA's are discussed later using preliminary flight trials with the test equipment in the flight test. 


\section{METHODOLOGY - RELATIVE NAVIGATION}

The calculated position and velocity estimates are not only used to navigate the UAS or manned aircraft, but also as an input to the ADS-B system and the FLARM. Furthermore, the datalink can be used to experiment with new relative navigation concepts developed by the researchers such as raw-measurement-based ADS-B [5]. In that approach both participants exchange Global Navigation Satellite System (GNSS) measurements instead of aircraft state vectors and performance parameters. Advantages of this method include: exploitation of well-established pseudorange double difference techniques, exploitation of well-established carrier-phase sequential difference techniques, improvement of the accuracy by the inherent removal of spatial and temporally correlated errors in double- and sequential differencing, increased observability by having both the measurements from the target and ownship, independence of a Datum Reference (e.g., coordinate frame is chosen by the sUAS using the measurement to calculate the position), accounts for correlations (both spatial and temporal), optimal weighting of the measurements in the position and velocity estimates, enables implementation of common GNSS integrity methods such as RAIM. Preliminary results based on earlier collected data, shows the performance that can be achieved in determining relative position and velocity (see Figure 3). The algorithms also allow the user to evaluate other relative navigation performance parameters (i.e. integrity, availability and continuity) so the safety of the mixed operations can be better evaluated. The basic approach is shown in Figure 4.

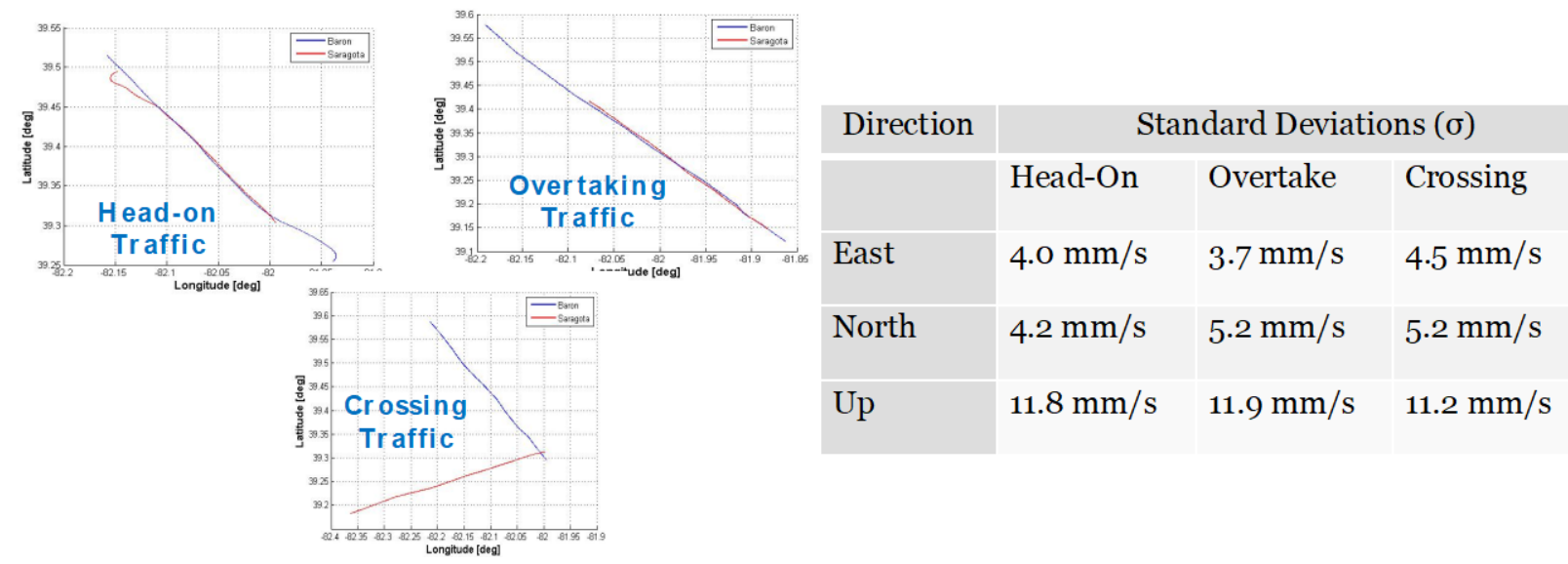

Fig. 3. Raw-measurement-based ADS-B as implemented as one of the surveillance sources in addition to ADS-B and FLARM. 


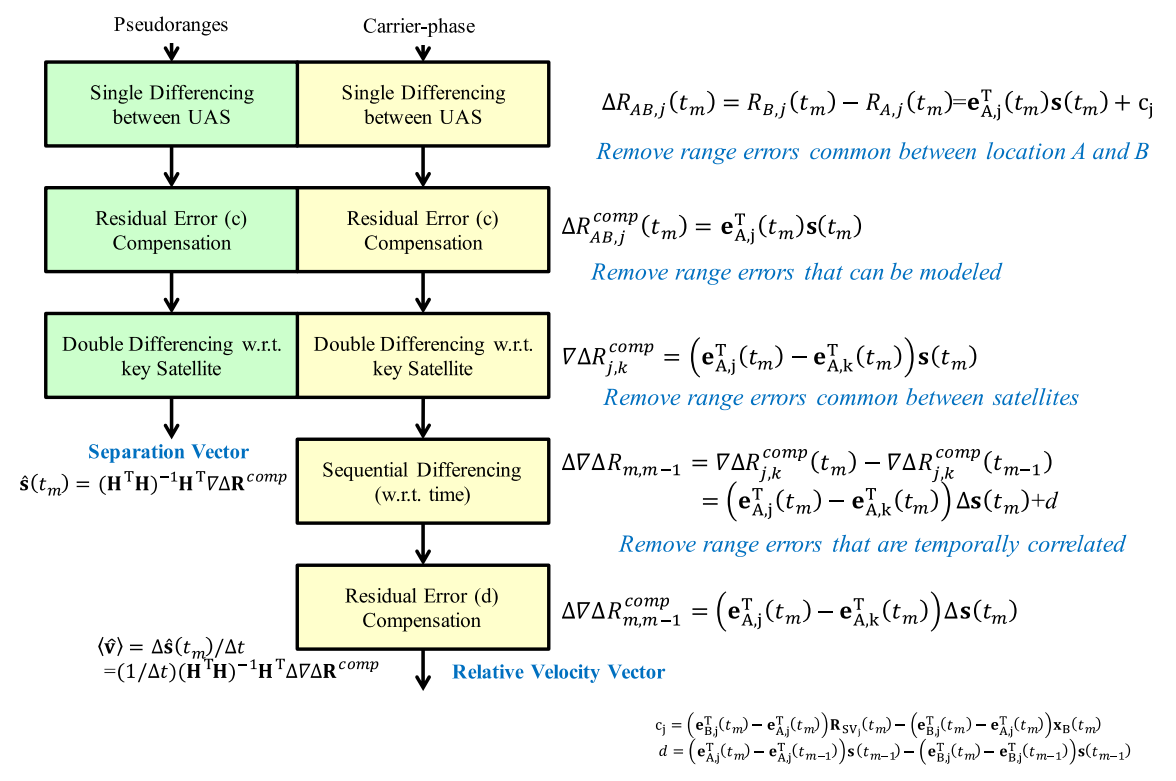

Fig. 4. Measurement-based relative navigation using GNSS pseudorange and carrier-phase measurements.

\section{EXPERIMENTAL SETUP}

The most important part of this paper is the experimental setup that has been prepared so future separation assurance methodologies can be evaluated for mixed airspace operations near airfields. The description of the experimental setup is divided into flight test aircraft and its scenario as well as the concepts to be reflected in the paper.

\subsection{Flight test aircraft}

With the LASER ultralight aircraft, the TUB has a universal test platform with numerous modifiable interfaces. The developed systems can be tested quickly and easily because complex certification procedures are not required in this aircraft class. The LASER serves as an additional vehicle for the navigation and surveillance platform and is an essential part of flight testing, validation, and verification trials. (i.e. self-separation of UAS to manned aircrafts). Figure 5 shows the LASER aircraft and its standard flight deck instrumentation. Future test may include custom-build Human Machine Interfaces (HMI) on the co-pilot side. 


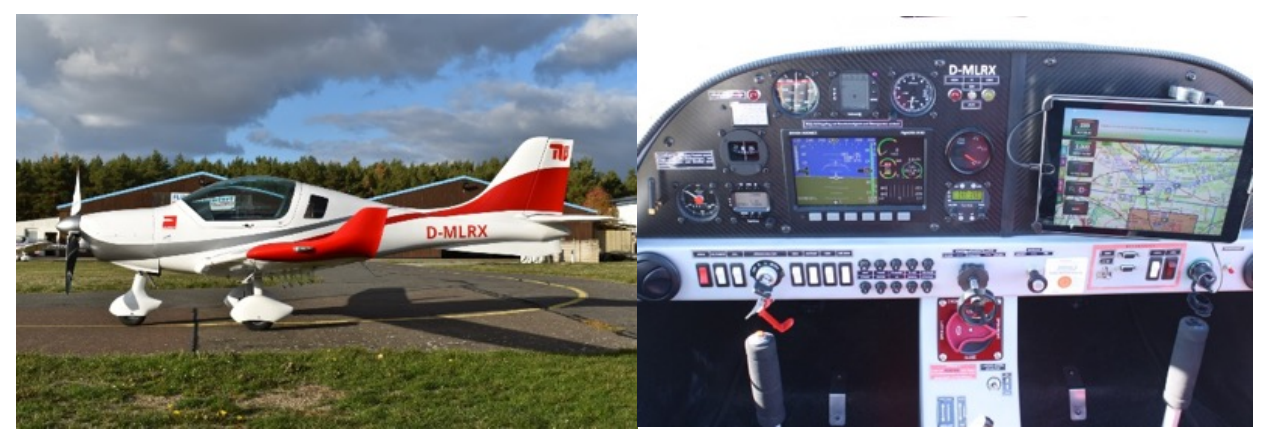

Figure 5. TU Berlin-owned ultralight aircraft LASER and it's flight deck

\subsection{Flight test scenario}

A scenario was defined to create multiple potential hazardous conflict situations while performing an operational pattern at the EDAV airfield. Figure 6 gives an overview of the general setup at the airfield. Basically, TU Berlin's LASER performed the standard left-hand traffic pattern on runway 28 with a visual approach (1) on a $3^{\circ}$-slope. In area (2) a car (imitating a UAS) was used to gather data with higher accelerations via a GNSS/SBAS receiver (U-blox ZED-F9T) or GNSS only receiver (U-blox ZED-F9P) mounted to the car's rooftop. The moving pattern of the car can be identified from Figure 8 (a). From the same figure (portion b) the movement in area (3) can be derived. In area (3), a slow-moving UAS pattern was simulated by walking members of the team using the exact same GNSS receivers. More detail will be discussed later. The displayed area (4) is the aircraft base and also hosted the deployed ground station and aircraft base.

An overview of the data links between the participants and areas are shown in Figure 7. The data sets collected for this paper, are position reports (generated by the EFIS) directly transmitted by LASER (1) via transponder and data in the raw ADS-B format [5]. Processed data set from the aircraft's EFIS FlightDEK-D180 collected data (3D positions) is extracted after the flights from software interacting with EFIS. Furthermore, a separate and aircraft-independent processor collects inflight GNSS data, which is transmitted (NMEA format) live to the ground station (4) using $\mathrm{XBee}$ data radio modules as corresponding equipment.

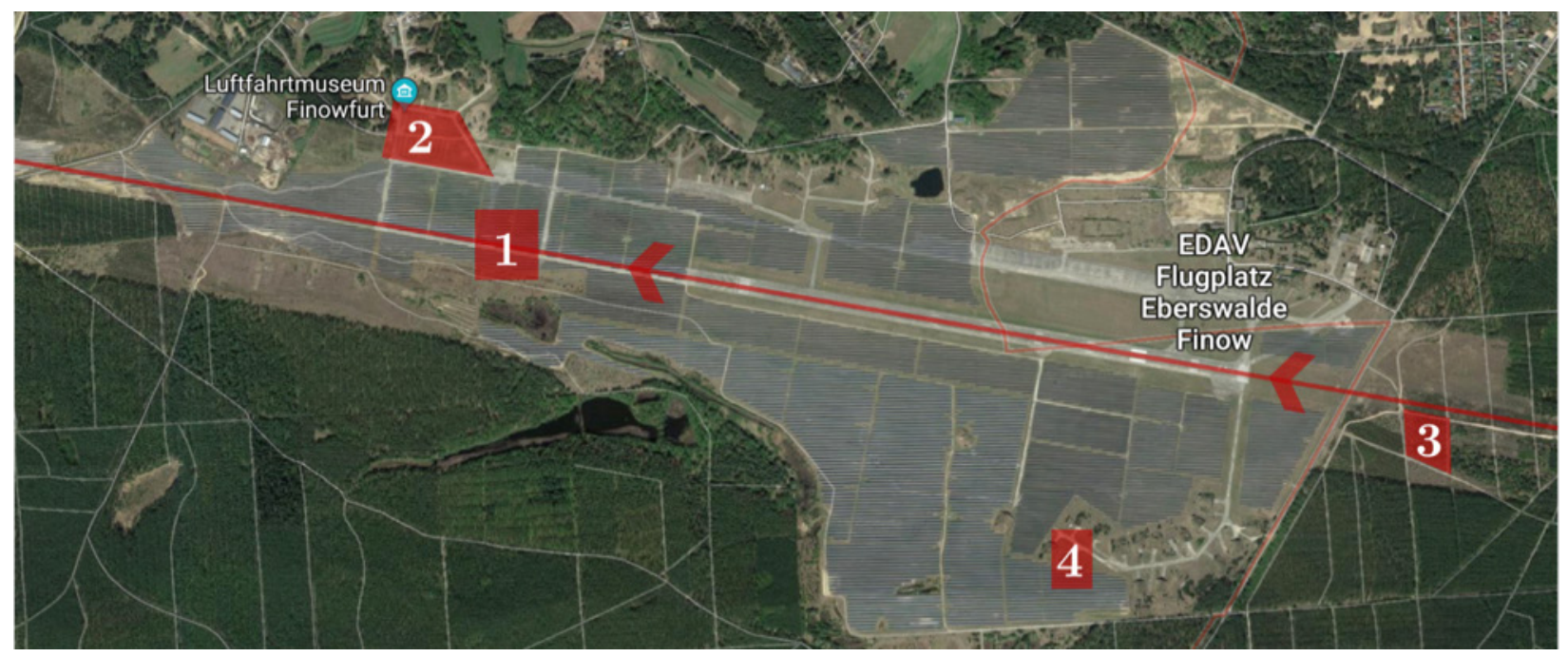

Figure 6. Flight Test Scenario at EDAV 


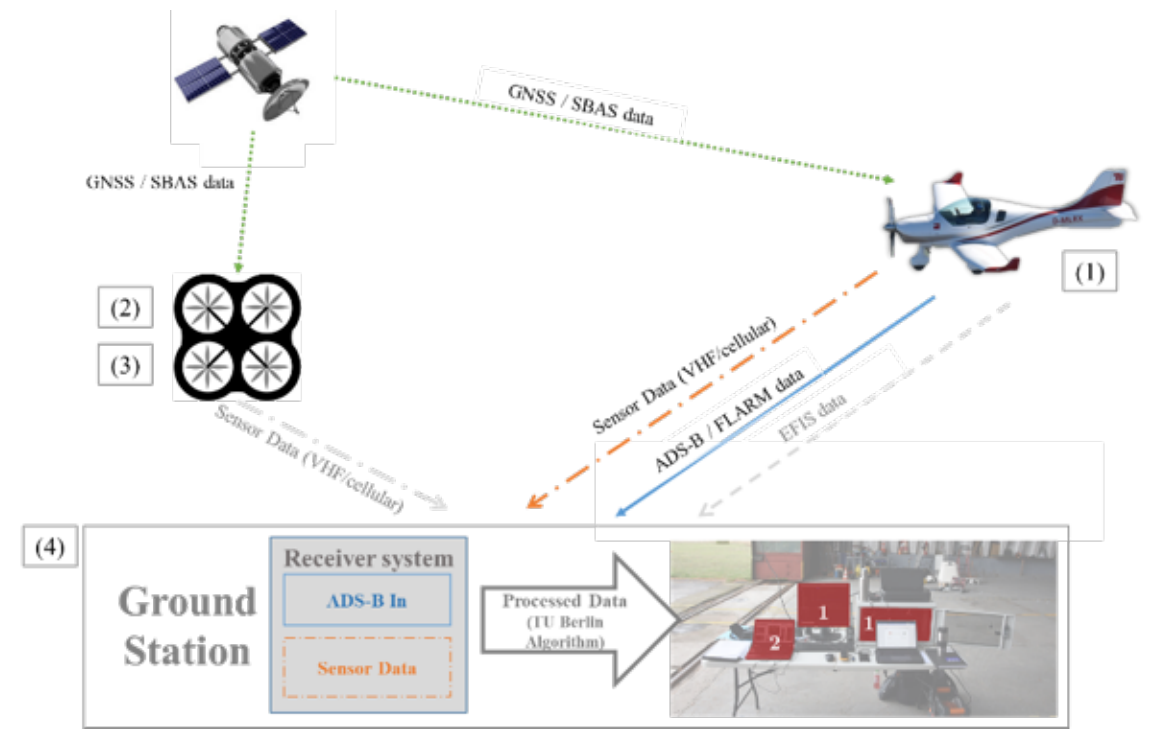

Fig.7. Data link setup for flight tests

The connection of the car (2) and walking team (3), depicted here as imitated UAS, is not yet upand-running. So, these "grey" data sets are extracted post flight test.

Figure 8 shows, in more detail, the above-mentioned participant trajectories, i.e. the trajectories of the simulated UAS with respect to the LASER creating the potential conflict scenarios. The two GNSS receivers are moved in their respective areas to create independent conflicts both parallel (2) and perpendicular/impinging (3) to LASER's flight direction. When the LASER passes predefined point-of-interest $(\mathrm{POI})$ the potential hazardous situation was built-up with the movement of the two "UAS" participants.
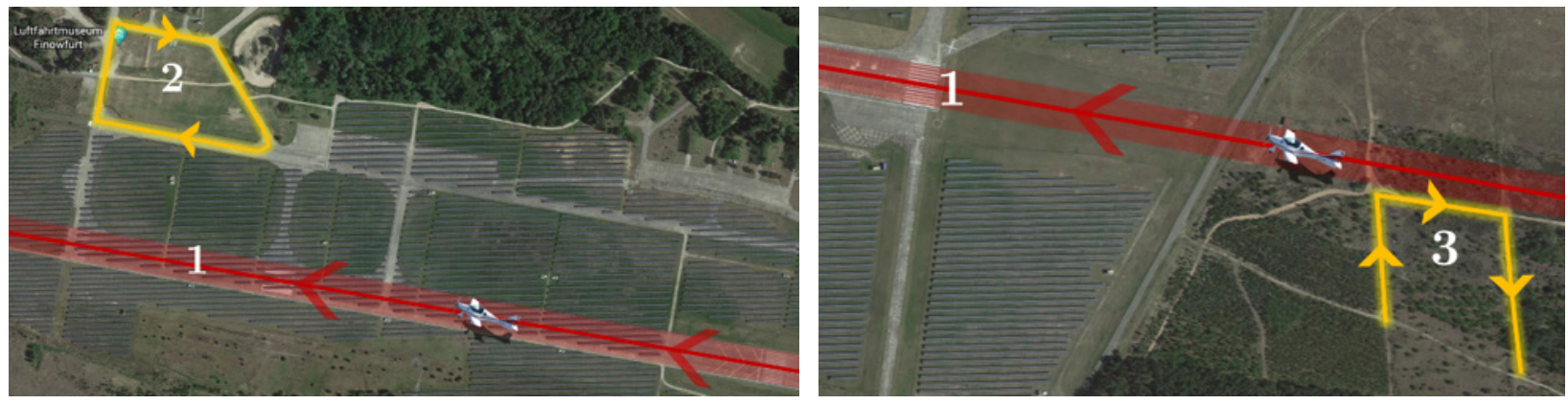

Fig. 8. Scenario initiating parallel (left; a) and obliging (right; b) movement

The parallel movement (2) was operated with a lateral separation of $>190 \mathrm{~m}$. Movement (3) on the other hand site was performed with a lateral path deviation of about $22 \mathrm{~m}$.

Lastly, for the flight test, the ground station is set up in area 4 (see Figure 6) with Visual Line of Sight (VLOS) to the LASER at flyovers of the runway and on downwind. The ground station consists of an ADS-B decoding interface filtering the LASER position reports using a TU Berlin developed code based on [6]. Furthermore, the XBee-connection to the LASER is based here and returns a live data connection. 


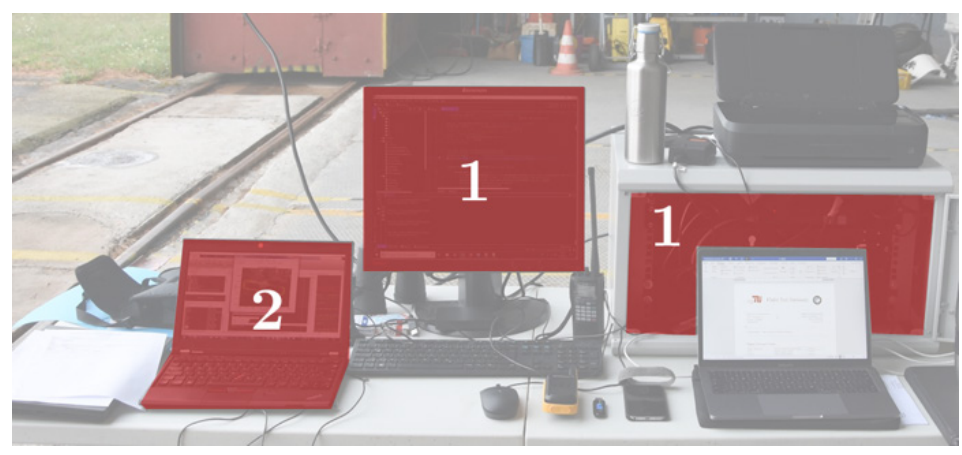

Fig. 9. Implementation of the ground station setup

Figure 9 shows the equipment implemented for ADS-B raw measurement decoding (1) and postprocessing unit (2) of gathered raw GNSS inflight data. The post-processing was performed with a TU Berlin developed code assessing the data sources with regards to accuracy, integrity, availability, continuity.

\subsection{Concept}

Before the actual flight tests took place in October and November 2019 a safety analysis with respect to possible unknown situations implemented through UAS operation in the close vicinity of a manned ultralight aircraft was performed. To determine such situations three different concept scenarios of general operation were developed. These concepts are distinguished according to their predictability, i.e. the level of a prior knowledge that is available regarding their planned trajectory, for operators of manned aircraft as well as their impact on operational safety. The following figures display the different concepts adopted to the operation at EDAV. Figure 10 shows the first concept with the least predictability. Here, the UL operation for the respective timestamps ( $T=0$ to $T=4$ ) is colored red with flight direction from East to the West (landing at EDAVs runway 28 on left hand site of the figure) following standard visual approach pattern. The walking team (imitating a slowly moving UAS) operates as shown in the yellow graph from starting point $\left(\mathrm{DP}_{\mathrm{T}=1}\right)$ with significant gain in altitude and change in position, both, to the current data point (DP $\left.\mathrm{T}_{\mathrm{T}=2}\right)$. For simplification the walking team's data points with timestamps $T=0$ and $T=4$ are left out, since the critical uncertain scenario takes place within the displayed timestep.

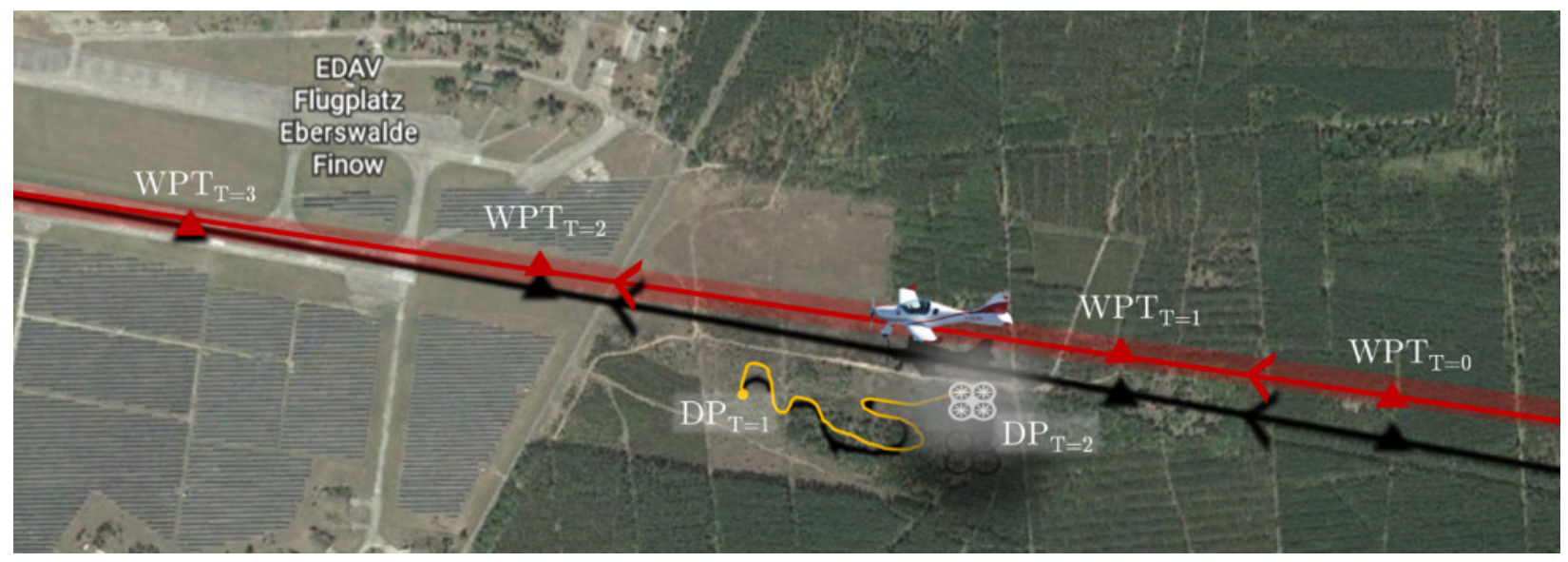

Fig. 10. Conceptual overview of a manned aircraft with a free-moving UAS operation in final approach 
This directly leads to a high risk in operational safety of the UL as well as the overall situation. Conceptually a constant need for position monitoring of the UAS and the UL is created to ensure

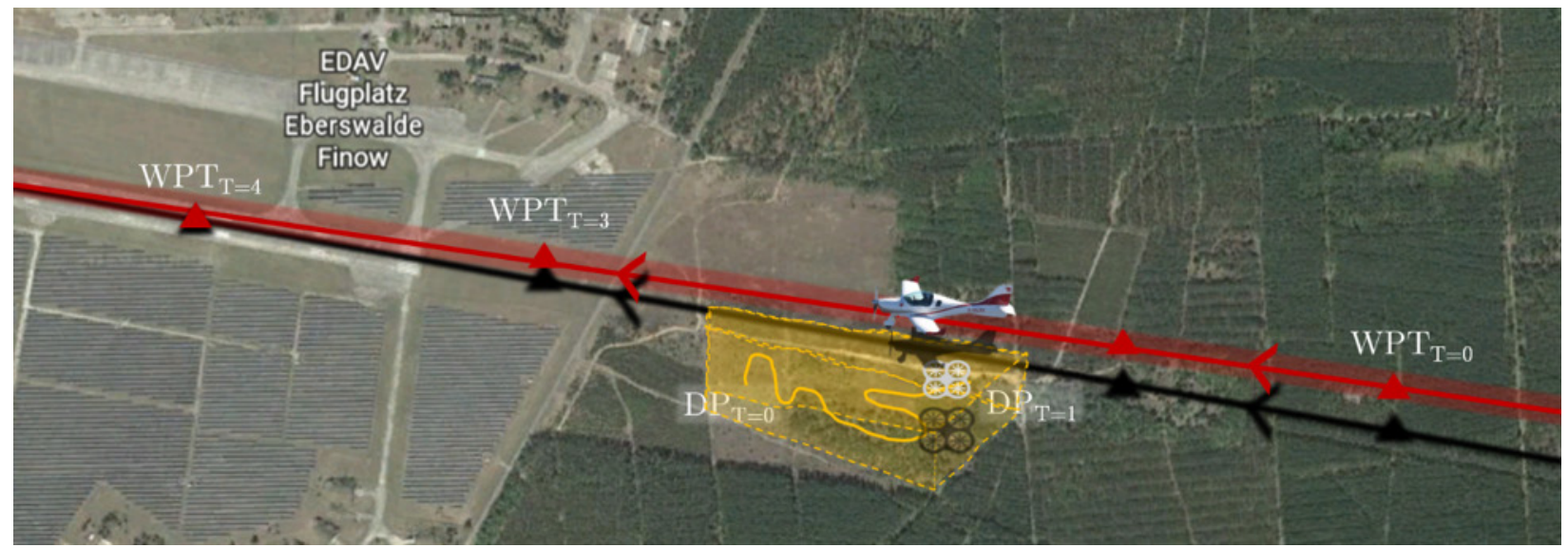

Fig. 11. Conceptual overview of a manned aircraft with a geo-fenced UAV operation in final approach

safe flight. Thus, the continuous estimation of the CPA of the objects tracks is necessary and implemented. Now, based on the violation of assessed criteria (distance or time to CPA) a defined countermeasure should be initiated on the UAS side to avoid a hazardous situation. This can be achieved by a proactive change in speed and/or bearing so that the protected airspace of the UL stays clear of intruders. In this case the prediction of the UAS flight path and resolve possible collision scenarios are probabilistic [7], geometric [8] or optimization-based [9]. Partially they rely on a additional onboard visual system that identifies a hazardous situation and, thus, counteract to resolve the situation.

The second concept predefines an 3D area, in which the UAS is allowed to move freely (yellowshaded area). Figure 11 visualizes the concept. As in the previous scenario, the track of the UL and the UAS remains the same. Since the operation of the LASER, in general, is located in a certain corridor around the airfield. The track of the UAS, holistically speaking, must be considered as unpredictable and arbitrary from the LASER point of view. Unlike Concept 1 the operation of the UAS is virtually geofenced, resulting in a lower risk of predictability and, thus, violation of separation minima, since this can be interpreted as an additional safety feature. Nevertheless, the calculation of (a) the constant monitoring in CPA of UL and UAS is to be implemented also to assure said separation minima and (b) the compliance of the UAS with the geofence must be monitored. In this concept, the geofenced airspace is reserved for UAS operation and does not allow time-wise airspace adoption nor passing of other air vehicles, manned or unmanned. Furthermore, geofence systems, used in today's UAS, often implement critical failure mode (i.e. SPOF) due to lacking independence of the geofence system and the UAS operating system (autopilot) as they both rely on GNSS signals. The concept can be understood as proposed by [10], where multiple static boundaries around an operating UAS are set up and constantly monitored. Furthermore, Dill et. al. tested a UAS-integrated protective solution in case of geofence violation. The focus of the present paper does not focus on this topic.

Another approach performed by [11] leads the way to a dynamic geofence, which changes based various vehicle parameters (speed etc.) and the wind component. This results in significantly 
smaller protected areas around the respective UAS. Other scientific work focusses on the violation of specific geofences. So, [12] et. al use the closure rate to a boundary to control violation/nonviolation of it. This can directly be transferred to the use case of the present work.

Compared to this approach, the third concept exploits the knowledge of a 3D or 4D (e.g. time) predefined UAS trajectory (e.g. using waypoints) similar to RNAV or, better, RNP where stringent performance and timing requirement must be met and non-conformance with this trajectory must be monitored. Figure 12 shows a possible and critical setup. The UAS fulfills a rectangular flight path along four Waypoints (WPTs), which are reached at generic timestamps 0 to 3, creating the actual hazardous scenario within timestep 1 and 2 . This possibly leads to an conflict situation with the LASER, since their respective flight path vectors are parallel with a minimum slant distance. Thus, concept 3 enhances the latter concept such that the restriction of an air column is not needed, but a corridor around the flight path has to be assured, which, basically, can be interpreted as RNP requirements for the UAS operation. If the UAS deviates from its intended path, this must be detection fast, and therefore, accurate knowledge and a high integrity of the velocity vector is a necessity. The raw measurement-based ADS-B concept supports this concept. Note, that it may be required to use a datalink other than the ADS-B one, as ADS-B, as is, may not be feasible for UAS.

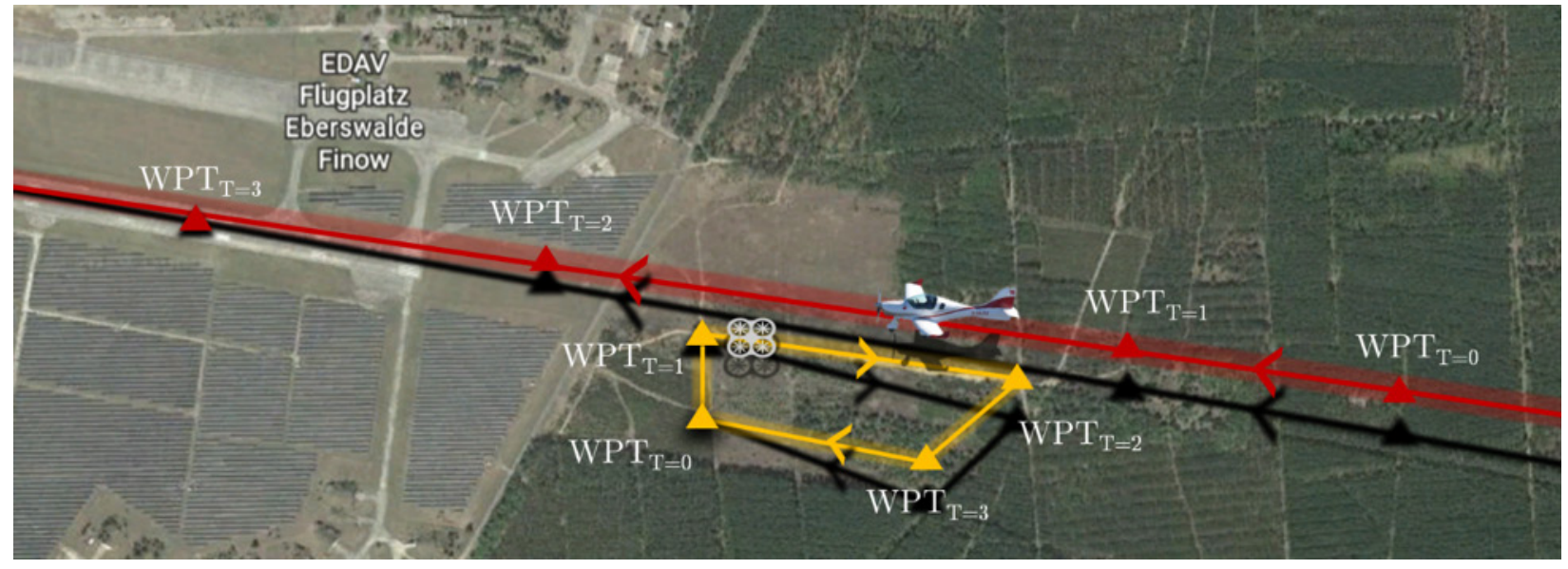

Fig. 12. Conceptual overview of a manned aircraft and an UAS operation in final approach with active Flight plan issued

Summarizing, the CPA-calculation will be part of the constituent concepts. The deviating parameters can be narrowed down to the predictability and conformance of the UAS movement towards the LASER's flight path assuring a minimum separation logic in this generic traffic distribution among manned and unmanned air vehicles.

For a broader understanding of the flight test results there are several influences, which must be considered. [13] refers to problems with using radio frequency-based navigation systems, since its accuracy is limited due to problems like multipath, signal attenuation, and shadowing. 


\section{PRELIMINARY RESULTS}

Flights were performed on three separate occasions in October and November 2019 in EDAV. The basic trajectories as calculated by GNSS are shown in Figure 14 on top of a Google map of the environment. Clearly, area (3) can be observed where the UAS is performing operations in the vicinity of the approach path of the LASER. The separation vectors between the LASER and UAS, the LASER and the ground station, and the UAS and the ground station are shown in Figure 14. The accuracy of the separation vectors, $\mathbf{s}_{i j}$, is better than that of the GNSS trajectories as spatially correlated errors are mitigates in the singe- and double-difference implementation to find the relative position vectors due to the method explained in Figure 4. The zoomed in plot on the righthand side of Figure 14 shows one of the dangerous situations developing when the LASER and UAS operate in close vicinity. The relative velocity, $\mathbf{v}_{i j}$, is shown in Figure 15.
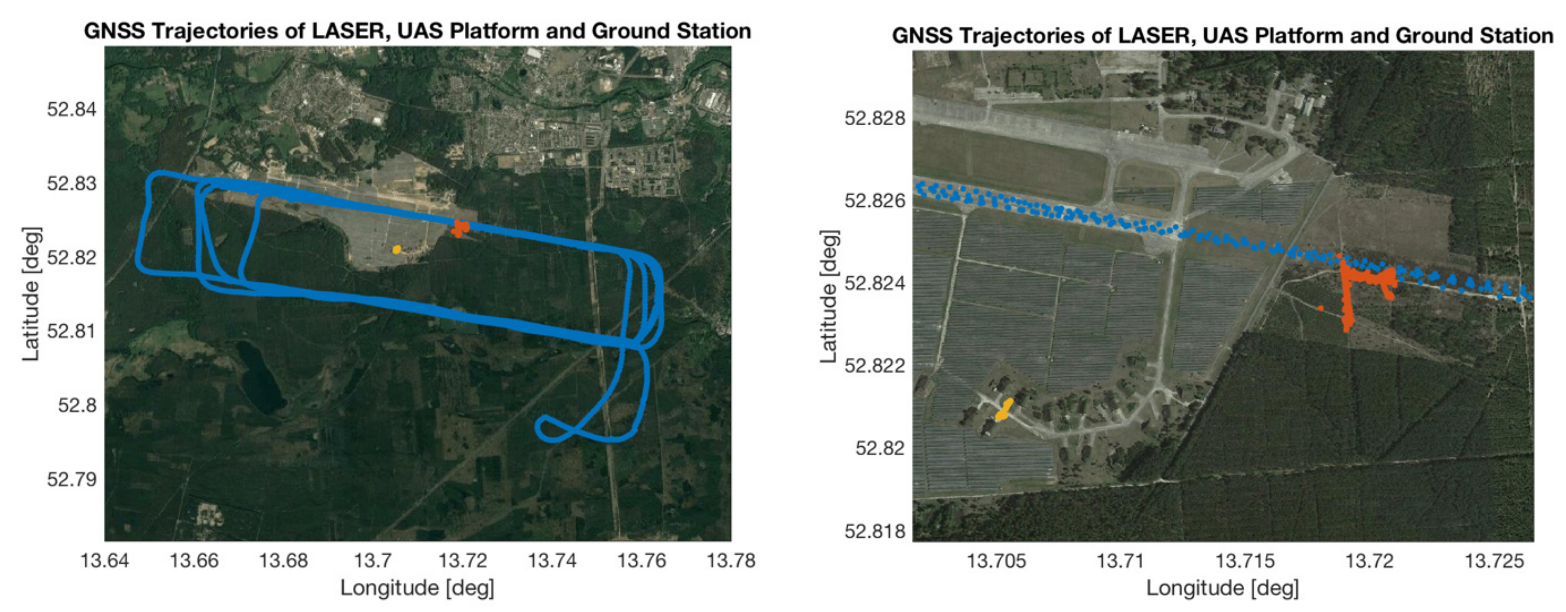

Fig. 13. GNSS Trajectories of the LASER Ultralight (UL), the simulated UAS, and ground station.
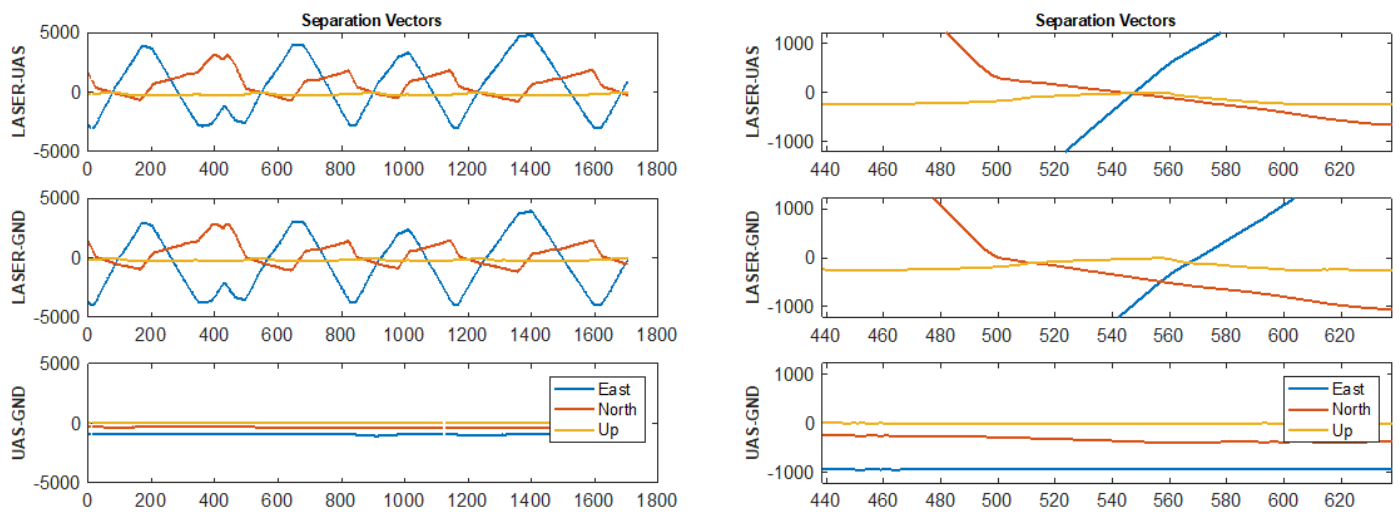

Fig. 14. Separation vectors for LASER-UAS, LASER-ground station and UAS-ground station. 

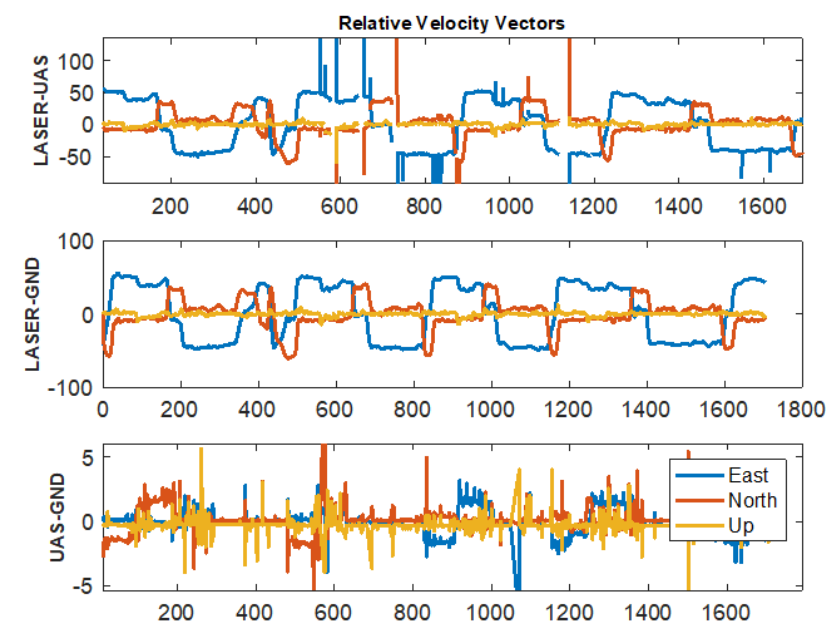

Fig. 15. Relative velocity vector.

The outliers and noise-like behavior in Figure 15 is due to the "simulated" behavior of the UAS where the antenna is basically carried by a member of the research team causing sometime large accelerations and thus velocities. Based on previous experience [14], the UAS motion is significant smoother even if operated manually. To get an idea of what the horizontal miss distance and $\tau$ value look like, the were computed using the separation and relative velocity vectors. The results are shown in Figure 16.
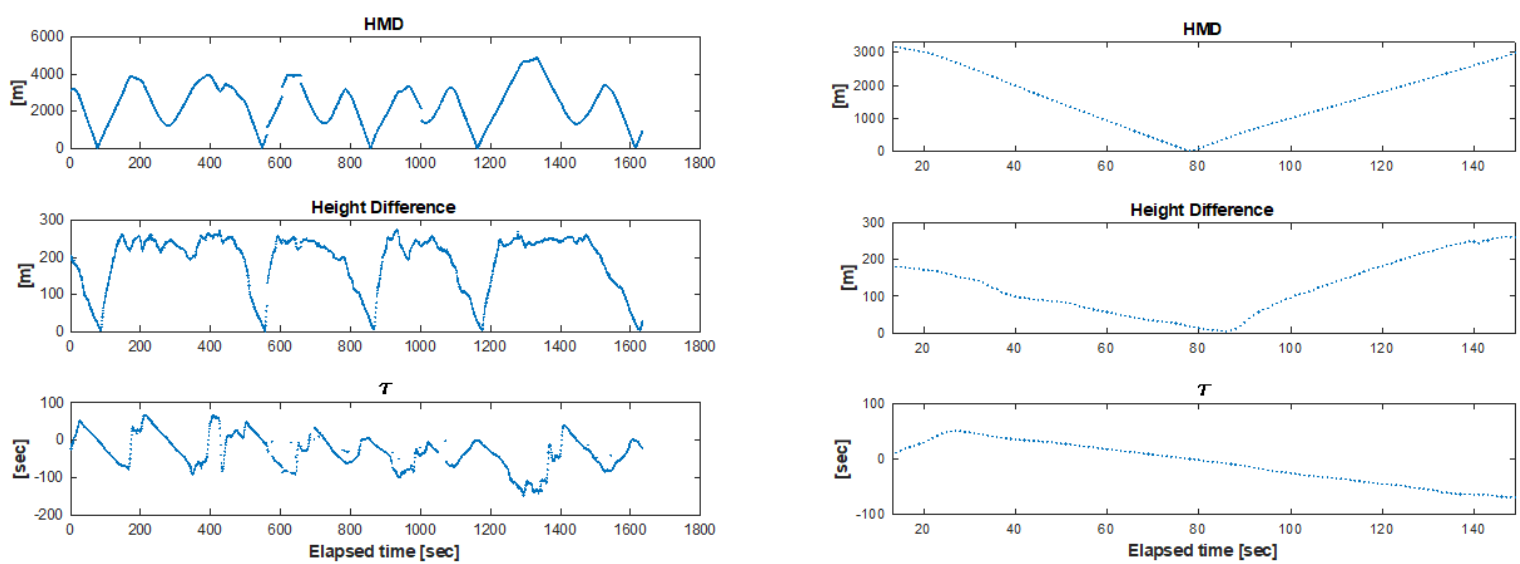

Fig. 16. Horizontal Miss Distance, Altitude Difference and $\tau$ for LASER-UAS operations.

Note that all three parameters are getting very small, well beyond the thresholds currently defined by TCAS or DAA. This is, of course, expected as in both TCAS and DAA, the UAS and aircraft are not operating intentionally at such small distances. The data here show some initial analysis and stress the importance of both further analyses in quantifying safe operations using the three concepts and the importance of a flight test facility. 


\section{SUMMARY AND CONCLUSIONS}

This paper discusses the development of an initial concept to safely operate general aviation aircraft and UAS simultaneously at low altitudes in the vicinity of an airfield and shows flight test results of a precise relative navigation and separation assurance system to support these operations. In the present work multiple hazardous concepts are established upfront. In the flight test setup these concepts were set up and initial data collected for analysis. All flight procedures were implemented successful and the data collection components were operating sufficiently, however, issues exist with the datalink that must be resolved in future flight trails. The outcomes of the preliminary work are (i) three concepts for mixed aircraft operations, (ii) a research airfield and flight test setup equipped to support future concept evaluations using flight tests with multiple UAS

and aircraft, (iii) a database with data that can be used by third parties for technology evaluation purposes.

\section{OUTLOOK}

For future flight test TU Berlin's researchers plan to establish improvements in three areas. These are (i) of technical, (ii) organizational and (iii) regulatory nature.

Corrections of (i) are seen in the setup of a stable live data link from all used air vehicles to ground station as proposed in Figure 17 with cellular and/or VHF communication. With the assurance of data links that incorporates the extension for FLARM as another conflict detection system. FLARM will support the LASER's situational awareness as well as supports decision-making at ground station as all operator-provided data are merged there. Furthermore, the ground station will assess the gathered data automatically with regards to hazardous interrogation scenarios and returns resolving recommendations based on geometric strategies. In case of the UAS operation this portion of communication will be using MAVLink format.

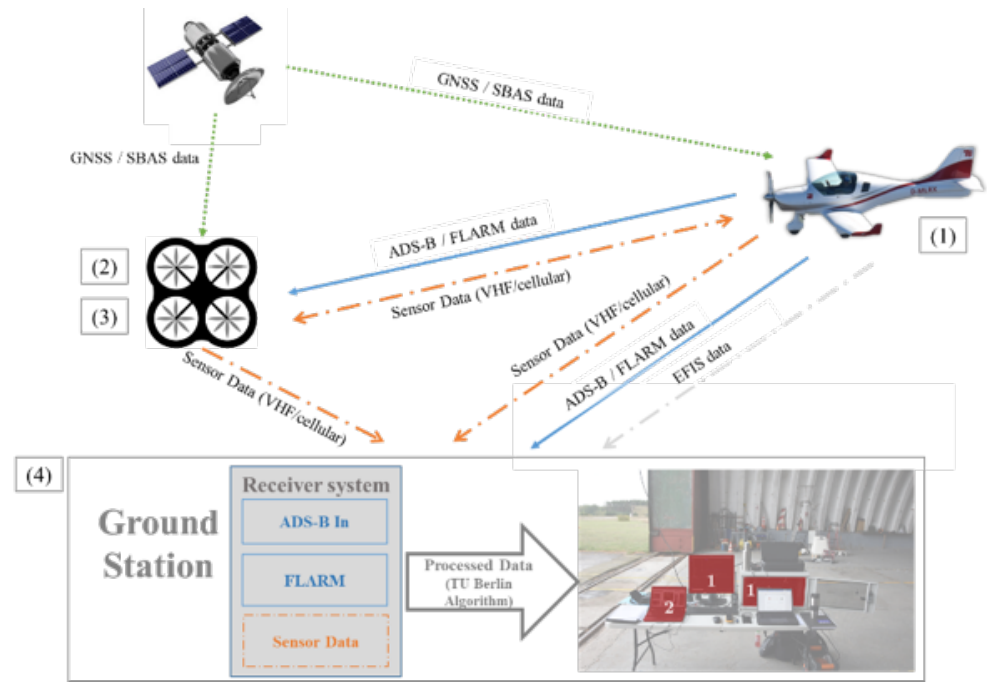

Fig. 17. Future flight test data link connections

To reach this goal, the hardware used in the flight tests will be re-evaluated for performance. Due to technical issues corresponding with limited data link range. Additionally, with this hardware 
setup in coherence with the shadowing through the aircrafts CFRP construction method, the antenna position will be re-arranged to the bottom of the fuselage. That leads to constructive changes in the LASER, an improvement on precipitation is to be expected.

Area (ii) incorporates the substitution of the UAS-imitating teams for physical UAS operating the missions. Which leads to improvement of area (iii), which can be derived from time-restricted legal approval of operation in the vicinity of an airfield as well as the vicinity of the protected areas around the flight path of an approaching aircraft for flight test purposes.

Finally, integrity equations will be developed for the algorithms developed in [5], so that the safety of the procedure can be addressed in eth context of all three concepts.

\section{REFERENCES}

[1] e. a. C. Munoz, DAIDALUS: Detect and Avoid Alerting Logic for Unmanned Systems, 2015 IEEE/AIAA 34th Digital Avionics Systems Conference (DASC), 2015.

[2] A. V. Nebylov, Aerospace Sensors, New York: Momentum Press, 2013.

[3] A. N. J. C. Munoz, A TCAS-II resolution advisory detection algorithm, AIAA Guidance, Navigation, and Control (GNC) Conference, 2013.

[4] SC-228, Minimum Operational Performance Standards (MOPS) for Detect and Avoid (DAA) Systems, RTCA, 2017.

[5] P. Duan und M. Uijt de Haag, Flight Test Results of a Measurement-Based ADS-B System for Separation Assurance, Bde. \%1 von \%2 NAVIGATION, Volume 60, Number 3, Fall 2013, , pp. pp. 221-234..

[6] J. Sun, The 1090MHz Riddle, 2017: GNU GPL v2 Open-Source License..

[7] K.-Y. \&. P. J.-W. \&. T. M.-J. Kim, „UAV collision avoidance using probabilistic method in 3D, 2007.

[8] S. K. Hong und H. L. N. Ngoc Thanh, „Completion of Collision Avoidance Control Algorithm for Multicopters," Seoul, South Korea, 2018.

[9] R. D. Alexander, J. A. McDermid und X. Zou, „On the Validation of a UAV Collision Avoidance System Developed by Model-Based Optimization: : Challenges and a Tentative Partial Solution," 2016.

[10] E. T. Dill und Y. S. D. K. J. , Safeguard - An Assured Safety Net Technology for UAS, N. L. R. Center, Hrsg., Hampton, Virginia, 2016. 
[11] S. D'Souza, A. K. Ishihara, B. E. Nikaido und H. Hasseeb, „Feasibility of Varying GeoFence Around an Unmanned Aircraft Operation Based on Vehicle Performance and Wind,“ 2016.

[12] S. Balachandran, A. Narkawicz, C. Munoz und M. Consiglio, „A Geofence Violation Prevention Mechanism for Small UAS, “ Belo Horizonte, Brazil, 2018.

[13] P. J. Teunissen und O. Montenbruck, Springer Handbook of Global Navigation Satellite Systems, Springer International Publishing Switzerland 2017, 2017.

[14] M. U. d. H. J. Huff, „Navigation of Multiple sUAS using GNSS, Inertial and Range Radios,“ in Proceedings of the ION Pacific Position, Navigation and Timekeeping, Waikiki, HI, 2019.

[15] A. Puri, Statistical Profile Generation of Real-time UAVbased Traffic Data, Tampa, FL: University of South Florida, 2008. 